\title{
PHLEBOTOMY WITH CONTROLLED HYPOVOLEMIA IS ASSOCIATED WITH DECREASED RED BLOOD CELL TRANSFUSION IN LIVER SURGERY
}

\section{Baker ${ }^{1,2}$, S. Bennett ${ }^{1,2}$, B. Chen ${ }^{1}$, S. Saeed ${ }^{1,2}$, C. WherRetT ${ }^{3}$, K. Bertens ${ }^{1}$, F.K. BalaA ${ }^{1}$, G. Martel ${ }^{1,2}$}

${ }^{1}$ Liver And Pancreas Unit, Department Of Surgery, University Of Ottawa. ${ }^{2}$ Clinical Epidemiology Program, Ottawa Hospital Research Institute. ${ }^{3}$ Department Of Anesthesiology, University Of Ottawa.

\section{INTRODUCTION}

- Blood loss during liver surgery is common $\rightarrow$ associated with worse perioperative morbidity and mortality

- Transfusion associated with significant risk

- Low central venous pressure (CVP) during liver transection protective of blood loss and transfusion requirement

- Intraoperative hypovolemic phlebotomy to lower CVP is associated with decreased blood loss

- The relationship between phlebotomy and transfusion requirement has yet to be determined

\section{OBJECTIVE}

Determine if hypovolemic phlebotomy is protective of red blood cell transfusion in patients undergoing liver resection
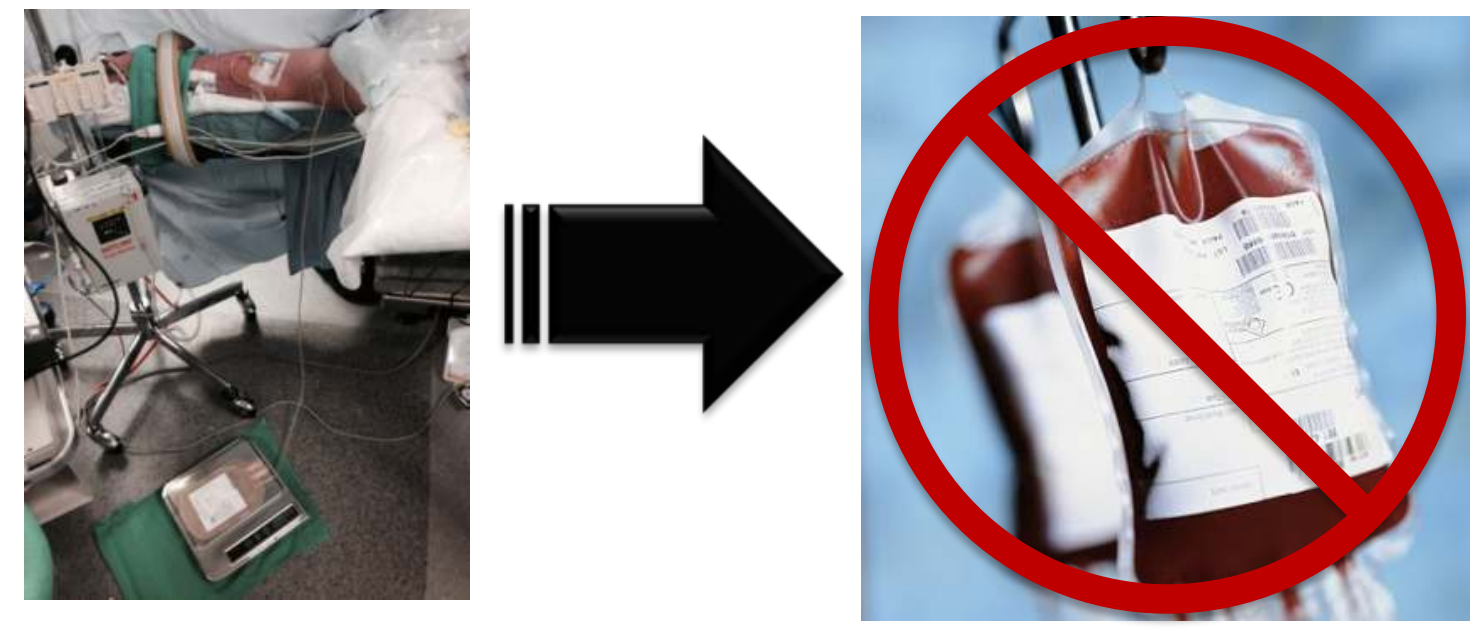

\section{METHODS}

Study design: retrospective review of prospectively collected data, cohort study: hypovolemic phlebotomy compared to control

Patient selection: all patients undergoing liver resection (2010-2016) at one referral center

\section{Phlebotomy technique:}

- Introduced in 2013, performed by one anesthetist

- $7-10 \mathrm{~mL} / \mathrm{kg}$ of patient body weight of whole blood removed in the 30 minutes preceding initiation of liver parenchymal transection Example: for a $70 \mathrm{~kg}$ patient, $490-700 \mathrm{~mL}$ whole blood removed

- Minimal replacement IV fluid administered, vasopressors preferentially used to maintain hemodynamics

- Blood retransfused following parenchymal transection

- If transfusion required, retransfused phlebotomized blood, followed by allogenic blood transfusion if necessary

Phlebotomized cohort $(n=45)$ :

- Intervention based on availability of anesthetist trained in the study protocol and offered to selected patients

- Patients agreeable, verbal consent obtained

- Major resections favored

- Patients with significant renal, cardiac or cerebrovascular disease were excluded

- Patients with anemia $(\mathrm{Hb}<100 \mathrm{~g} / \mathrm{L})$, coagulopathy $(\mathrm{INR}>1.5$ or platelets $<100 \times 10^{\wedge} 9 / \mathrm{L}$ ) or hepatic insufficiency (bilirubin $>35 \mathrm{umol} / \mathrm{L}$ ) excluded

Data collected: Patient demographics, surgical details, laboratory data, phlebotomy data, and transfusion data were retrieved.

\begin{tabular}{|ll|}
\hline & Patient factors \\
\hline - & Gender \\
- & BMI \\
- & PAD \\
- & Hb $\mathbf{b}<\mathbf{1 2 5}$ \\
- & Hb value \\
- & Platelet value \\
\hline
\end{tabular}
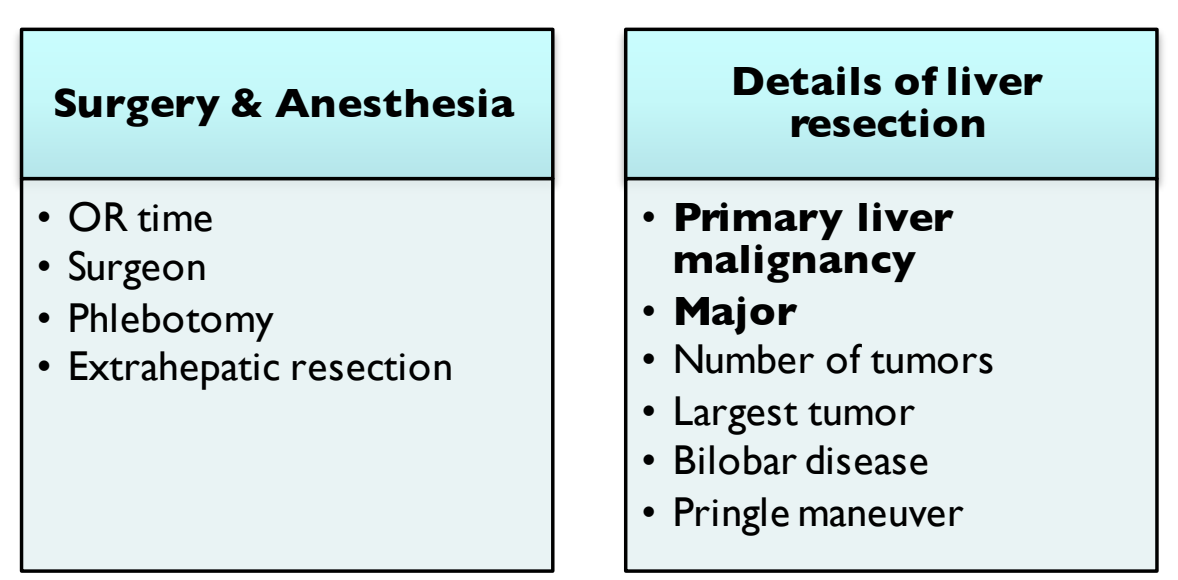

Statistical analysis: Variables identified on univariate regression associated with transfusion ( $p \leq 0.2$ ) examined in a multivariate logistic regression model

\section{RESULTS}

Table 1. A significantly larger proportion of patients underwent major hepatic resections and resections for primary liver malignancy in the phlebotomized cohort $(p<0.05)$

\begin{tabular}{|c|c|c|c|}
\hline & $\begin{array}{l}\text { Phlebotomy } \\
(n=45)\end{array}$ & $\begin{array}{l}\text { Control } \\
(n=328)\end{array}$ & p \\
\hline Age, median & $\begin{array}{l}63.4(30.7, \\
84.3)\end{array}$ & $\begin{array}{l}63.8(21.8, \\
86.0)\end{array}$ & 0.578 \\
\hline Gender, female & $18(40.0 \%)$ & 167 (50.9\%) & 0.170 \\
\hline BMI, median & $\begin{array}{l}24.5(17.6, \\
43.9)\end{array}$ & $\begin{array}{l}27.5(15.3, \\
68.3)\end{array}$ & 0.0588 \\
\hline CAD & $4(8.9 \%)$ & $26(7.9 \%)$ & 0.772 \\
\hline Major resections & $38(84.4 \%)$ & $140(42.7 \%)$ & $<0.0001$ \\
\hline Indication & & & 0.0558 \\
\hline MCRC & $22(48.9 \%)$ & $167(51.1 \%)$ & \\
\hline Cholangio/GB & $6(13.3 \%)$ & $34(10.4 \%)$ & \\
\hline $\mathrm{HCC}$ & $8(17.8 \%)$ & $23(7.0 \%)$ & \\
\hline Other & $9(20.0 \%)$ & $103(31.5 \%)$ & \\
\hline Primary liver CA & $14(31.1 \%)$ & $57(17.4 \%)$ & 0.0278 \\
\hline
\end{tabular}

Table 2. Phlebotomy was associated with decreased blood loss and transfusion requirement $(p<0.05)$

\begin{tabular}{|c|c|c|c|}
\hline & $\begin{array}{l}\text { Phlebotomy } \\
(n=45)\end{array}$ & $\begin{array}{l}\text { Control } \\
(n=328)\end{array}$ & $p$ \\
\hline OR time, median & $305(180,526)$ & $284(62,666)$ & 0.473 \\
\hline Phlebotomy, g, median & $521(260,850)$ & - & - \\
\hline $\begin{array}{l}\text { Phlebotomy, g/kg, } \\
\text { median }\end{array}$ & $7.23(4.7,10.2)$ & - & - \\
\hline Blood loss, median & $400(100,2100)$ & $500(0,12000)$ & 0.0563 \\
\hline $\begin{array}{l}\text { Blood loss, } \mathrm{mL} / \mathrm{kg} \text {, } \\
\text { median }\end{array}$ & $\begin{array}{l}5.87(1.42, \\
22.6)\end{array}$ & $6.73(0,122.4)$ & 0.0417 \\
\hline Losses $>1,000 \mathrm{~mL}$ & $8(17.8 \%)$ & $74(26.3 \%)$ & 0.219 \\
\hline \multicolumn{4}{|l|}{ pRBC Transfusion } \\
\hline Index admission & $5(11.1 \%)$ & $77(23.5 \%)$ & 0.0604 \\
\hline Intraoperative & $2(4.4 \%)$ & $49(15.4 \%)$ & 0.0476 \\
\hline Postoperative & $4(8.9 \%)$ & $53(16.7 \%)$ & 0.180 \\
\hline Perioperative 1 week & $4(8.9 \%)$ & $61(18.6 \%)$ & 0.107 \\
\hline Length of stay & $7(4,36)$ & $6(1,369)$ & 0.669 \\
\hline
\end{tabular}

Table 3. Predictors of blood transfusion by multivariate analysis. Phlebotomy group 4.3 times less likely to receive blood transfusion.

\begin{tabular}{|llll}
\hline Variables & OR & $\mathbf{9 5 \%}$ CI & $\mathbf{p}$ \\
\hline Phlebotomy & 0.231 & $0.081-0.655$ & 0.0059 \\
\hline Major resection & 2.858 & $1.622-5.036$ & 0.0003 \\
Preop hemoglobin $<12.5 \mathrm{~g} / \mathrm{dL}$ & 6.045 & $3.482-10.493$ & $<0.0001$
\end{tabular}

Variables included in multivariable model: phlebotomy, CAD, major liver resection, preoperative $\mathrm{Hb}<125$, extrahepatic resection

\section{CONCLUSION}

Phlebotomy with controlled hypovolemia appears to be significantly protective of red blood cell transfusion, independent from other known risk factors 